\title{
HARMONIC FUNCTIONS AND THEIR CONJUGATES ON HILBERT SPACE
}

\author{
BY \\ MICHAEL J. FISHER(')
}

Introduction. Let $1<p<\infty$ and let $H$ be a real separable Hilbert space. Denote by $y \rightarrow T_{y}$ the regular representation of the additive group of $H$ acting on $L^{p}$ ( $H$, normal distribution). Let $B$ be a one-one Hilbert-Schmidt operator on $H$ and $e_{1}, e_{2}, e_{3}, \ldots$ be an orthonormal basis for $H$. Let $n_{t}$ denote the normal distribution with variance parameter $t / 2$, let the function $n_{t}(z)=(\pi t)^{-1 / 2} \exp \left[-z^{2} / t\right]$, and let the function $N_{t}(z)=z n_{t}(z)$. Set

$$
P_{z}(f)=\int_{J}^{\infty} H_{t}(f) N_{t}(z) d t / t
$$

where

$$
H_{t}(f)=\int_{H} T_{y} f d n_{t} \circ B^{-1}(y)
$$

for $f$ in $L^{p}(H) . P_{z}(f)$ is the Poisson integral of $f$. The conjugate Poisson integrals of $f$ are the

$$
Q_{z}^{(j)}(f)=\lim _{R \rightarrow \infty} \int_{0}^{R}\left[\int T_{B y} f\left(y, e_{j}\right) d n_{t}(y)\right] n_{\iota}(z) d t / t .
$$

[At this point we can not specify the domain of integration for the inner integral.] $Q_{z}^{(j)}(f)$ is a type of singular integral operator and is bounded on $L^{p}(H)$. We shall show that $Q_{z}^{(j)}$ is the composition of the Poisson integral with a singular integral operator of the type discussed in [1].

Let $A_{j}$ denote the infinitesimal generator of the semigroup $T_{t B e_{j}}, t>0$. The $A_{j}$ will play the role of derivatives. $P_{z}(f)$ is infinitely differentiable for each $z>0$ and we have an integral formula for its derivatives. For $p=2,\left(\partial^{2} / \partial z^{2}\right) P_{z}(f)+\sum_{j=1}^{\infty} A_{j}^{2} P_{z}(f)$ $=0$ and, conversely, if $U(z)$ is a twice differentiable function from $(0, \infty)$ to $L^{2}(H)$ such that $\left(\partial^{2} / \partial z^{2}\right) U(z)+\sum_{j=1}^{\infty} A_{j}^{2} U(z)=0$ and $\|U(z)\|_{2}$ is bounded in $z>0$, there is a unique $f$ in $L^{2}(H)$ such that $U(z)=P_{z}(f)$.

For $f$ in $L^{2}(H)$, set $U(z)=P_{z}(f), v_{j}(z)=-Q_{z}^{(j)}(f), V(z)=\left(v_{1}(z), v_{2}(z), v_{3}(z), \ldots\right)$, and $F(z)=(U(z), V(z))$. Then $|F(z)|$ is in $L^{2}(H)$ for $z \geqq 0$ and the Cauchy-Riemann equations relate the components of $F$. If $|F(z)|>0$ almost everywhere for $z>0$, the sum $\left(\partial^{2} / \partial z^{2}\right)|F(z)|+\sum_{j=1}^{\infty} A_{j}^{2}|F(z)|$ converges in measure and the sum is positive

Presented to the Society, August 31, 1967; received by the editors November 21, 1967.

${ }^{(1)}$ Research supported by the Air Force Office of Scientific Research, Contract No. AF 49(638)-1382. 
almost everywhere. If $G(z)=\left((\partial / \partial z) U(z), A_{1} v_{1}(z), A_{2} v_{2}(z), A_{3} v_{3}(z), \ldots\right)$ and if $|G(z)|>0$ almost everywhere for $z>0$, the sum $\left(\partial^{2} / \partial z^{2}\right)|G(z)|+\sum_{j=1}^{\infty} A_{j}^{2}|G(z)|$ converges in measure and is positive almost everywhere.

When $H$ is a finite dimensional Euclidean space and $B$ is the identity operator on $H$ the Poisson integral and the conjugate Poisson integrals, given above, reduce to the classical finite dimensional forms of these operators. The results to be discussed in this paper correspond to known theorems on finite dimensional spaces; see Weiss and Stein [10] in this regard.

1. Preliminaries. To minimize the discussion of measure theory on Hilbert space we refer the reader to the papers [3] and [5] of L. Gross and [8] and [9] of I. E. Segal.

Definition (Segal). A weak distribution on a real Hilbert space, $H$, is an equivalence class of linear maps, $F$, from the conjugate space $H^{*}$ of $H$ to real valued measurable functions on a probability space (depending on $F$ ). Two such maps, $F$ and $F^{\prime}$, are equivalent if for any finite set of vectors $y_{1}, \ldots, y_{k}$ in $H^{*}$, $F\left(y_{1}\right), \ldots, F\left(y_{k}\right)$ and $F^{\prime}\left(y_{1}\right), \ldots, F^{\prime}\left(y_{k}\right)$ have the same joint distribution in $k$-space. A weak distribution is continuous if a representative is a continuous linear map (the range space has the topology of convergence in measure).

In what follows we shall be most interested in the normal distribution with variance parameter $c>0$. This distribution is uniquely determined by the following properties: (1) for any $y$ in $H^{*}, n(y)$ is normally distributed with mean zero and variance $c\|y\|^{2}$; (2) $n$ maps orthogonal vectors to independent random variables. The normal distribution is continuous. There is an essentially unique (up to expectation preserving isomorphism) probability space $(S, \Sigma, \mu)$ and a continuous linear map, $F$, from $H^{*}$ to the real valued measurable functions on $(S, \Sigma, \mu)$ such that $F$ is a representative of the normal distribution. $\Sigma$ has no proper sub- $\sigma$-field with respect to which all of the $F(y), y$ in $H^{*}$, are measurable. The measurable functions on $H$ are the measurable functions on $(S, \Sigma, \mu) . L^{p}\left(H, n_{c}\right)=L^{p}(S, \Sigma, \mu)$. When the variance parameter is 1 , we denote $n=n_{1}$ and $L^{p}(H)=L^{p}(H, n)$. The expectation, $E(f)$, of a measurable function $f$ is $E(f)=\int_{S} f d \mu$.

A function $f(x)$ on the points of $H$ is a tame function if there is a Baire function $g$ on a finite dimensional Euclidean space, $E_{k}$, and orthonormal vectors, $h_{1}, \ldots, h_{k}$ in $H^{*}$ such that $f(x)=g\left(\left(x, h_{1}\right), \ldots,\left(x, h_{k}\right)\right)$. The span of the $h_{i}, i=1, \ldots, k$ in $H$ is called the base space of $f$. If $F$ is a representative of the normal distribution and $f(x)=g\left(\left(x, h_{1}\right), \ldots,\left(x, h_{k}\right)\right)$ is a tame function $\tilde{f}(s)=g\left(F\left(h_{1}\right)(s), \ldots, F\left(h_{k}\right)(s)\right)$ is a measurable function on $H$. The expectation of $\tilde{f}$ is

$$
E(\tilde{f})=(2 \pi c)^{-k / 2} \int_{E_{k}} g(t) \exp \left[-\frac{\|t\|^{2}}{2 c}\right] d t,
$$

where $k$ is the dimension of the base space of $f$. This equality holds in the sense that if either side exists and is finite then so does the other and the two are equal.

Several very useful representatives of the normal distribution are known. Of 
these the one in which we shall be most interested is the mapping studied by Gross (in [5]) from $H^{*}$ to Borel measurable functions on an abstract Wiener space. We adopt the notation and terminology of [5]. Let $B$ be a one-one Hilbert-Schmidt operator on a real separable Hilbert space $H$. Then $\|B . x\|=|x|_{1}$ is a measurable norm on $H$. Let $H_{B}$ denote the completion of $H$ in this norm. Let $S$ denote the $\sigma$-field generated by the closed subsets of $H_{B}$. The normal distribution $n_{c}$ induces a Borel probability measure $N_{c}$ on $H_{B}$ such that the extension of the identity map on $H_{B}^{*}\left(\subset H^{*}\right)$, regarded as a densely defined map on $H^{*}$ to measurable functions on $\left(H_{B}, S, N_{c}\right)$, to $H^{*}$ is a representative of the normal distribution on $H$. Continuous functions, $f$, on $H_{B}$ are measurable functions on $H$ and if $g$ denotes the restriction of $f$ to $H$ and if $\mathscr{F}$ denotes the directed set (ordered by inclusion of the ranges) of finite dimensional projections on $H$, the net $\{\tilde{g}(Q x) \mid Q \in \mathscr{F}\}$ of measurable tame functions converges in measure to $f$ as $Q$ tends strongly to the identity through $\mathscr{F}$.

Let $N_{c}$ be as above. We may regard $B$ as an isometry from $H_{B}$ to $H$. Hence $N_{c} \circ B^{-1}$ is a Borel measure on $H$. This measure is usually denoted by $n_{c} \circ B^{-1}$. See [4] for a discussion of these measures. If $f$ is a bounded continuous function from $H$ to a Banach space $E, \int_{H} f(x) d n_{c} \circ B^{-1}(x)=\int_{H_{B}} f(B y) d N_{c}(y)=E(f \circ B)^{\sim}$.

If $f, g$, and $f g$ are absolutely integrable tame functions on $H,(f g)^{\sim}=\tilde{f} \tilde{g},(a f+g)^{\sim}$ $=a \tilde{f}+\tilde{g}$ for constants $a$, and if $f \leqq g$ on $H, \tilde{f} \leqq \tilde{g}$ almost everywhere. We shall use these properties often. Gross [3] has studied other functions on the points of $H$ which give rise to measurable functions on $H$. If $C$ is a Hilbert-Schmidt operator on $H$, $\|C x\|$ determines a measurable function $\|C(\cdot)\| \sim$ on $H$ by

$$
\|C(\cdot)\| \sim=\lim \{\|C Q x\| \sim \mid Q \in \mathscr{F}\}
$$

where $\mathscr{F}$ is as above and $Q$ tends strongly to the identity through $\mathscr{F}$. If $f$ is a bounded continuous function on $H, f(C x)$ determines a measurable function on $H$. Such elementary functions as $\|x\|, \exp \left[-\|x\|^{2}\right]$, and $\exp [i\|x\|]$ fail to determine measurable functions on $H$. We refer to [3] for further discussion of these points.

2. Harmonic functions. We shall be interested in functions $U:(0, \infty) \rightarrow L^{p}(H)$, $1<p<\infty$, which are twice strongly differentiable. Let $e_{1}, e_{2}, e_{3}, \ldots$, be an orthonormal basis for the real separable Hilbert space $H$. Let $B$ be a one-one HilbertSchmidt operator on $H$ and let $y \rightarrow T_{y}$ denote the regular representation of the additive group of $H$ acting on $L^{p}(H) .\left\{T_{y}\right\}$ is a strongly continuous group of isometries on $L^{p}(H)$; if $f$ is a bounded tame function $\left(T_{y} f\right)(x)=f(x-y) D_{p}(x, y)$ where $D_{p}(x, y)=\exp \left[(x, y) / p-\|y\|^{2} / 2 p\right]$. See [1]. Let $A_{j}$ denote the infinitesimal generator of the semigroup $T_{t B e}, t>0$. For any $h$ in $H$, let $A_{h}$ be the infinitesimal generator of the semigroup $T_{t B h}, t>0$. Each $A_{h}$ is a closed densely defined linear operator on $L^{p}(H)$. We say that $f$ in $L^{p}(H)$ is differentiable if $f$ is in the domain of $A_{h}$ for each $h$ in $H$. $f$ is twice differentiable if $A_{h} f$ exists for $h \in H$ and if $f$ and $A_{h} f$ are in the domain of $A_{k}$ for all $h$ and $k$ in $H$. $f$ is $n$-times differentiable if when $h_{1}, \ldots, h_{i}$ are vectors in $H$ and $\alpha=\left(\alpha_{1}, \ldots, \alpha_{i}\right)$ is a multi-index of order $|\alpha|=\sum_{j=1}^{i} \alpha_{j} \leqq n-1$ 
then $A^{\alpha} f=A_{h_{1}}^{\alpha 1} \cdots A_{h_{i}}^{\alpha i} f$ exists and is in the domain of $A_{h}$ for any $h$ in $H$. $f$ is infinitely differentiable if it is $n$-times differentiable for every $n$.

Let $U(z)$ be a twice strongly differentiable function from $(0, \infty)$ to $L^{p}(H)$ such that $U(z)$ is twice differentiable as a function in $L^{p}(H)$ for each $z>0$. Such a function will be said to be harmonic if for $z>0 U_{z z}(z)+\sum_{j=1}^{\infty} A_{j}^{2} U(z)=0$ where the sum converges in $L^{p}(H)$. In this section we shall characterize the harmonic functions $U:(0, \infty) \rightarrow L^{2}(H)$ for which $\|U(z)\|_{2}$ is a bounded function of $z$ for $z>0$.

Let $n_{t}$ denote the normal distribution with variance parameter $t / 2$, and let $n_{t} \circ B^{-1}$ denote the Gaussian measure with representative $F \circ B^{*}$ when $F$ is the representative of $n_{t}$, see [4]. For $f$ in $L^{p}(H)$ set $H_{t}(f)=\int_{H} T_{y} f d n_{t} \circ B^{-1}(y)$. Then $\left\|H_{t}(f)\right\|_{p} \leqq\|f\|_{p}$ for $t \geqq 0$. By taking Fourier transforms one sees that $\left(n_{t} \circ B^{-1}\right) *$ $\left(n_{s} \circ B^{-1}\right)=\left(n_{t+s} \circ B^{-1}\right)$, so that $H_{t}\left(H_{s}(f)\right)=H_{t+s}(f)$. Since

$$
H_{t}(f)=\int_{H} T_{t^{1 / 2} y} f d n_{1} \circ B^{-1}(y)
$$

it follows from the strong continuity of the $T_{y}$ and from the dominated convergence theorem that $\lim _{t \rightarrow 0^{+}} H_{t}(f)=f$. Thus $H_{t}$ is a strongly continuous contraction semigroup on $L^{p}(H)$.

Set

$$
n_{t}(z)=(\pi t)^{-1 / 2} \exp \left[-z^{2} / t\right], \quad N_{t}(z)=z n_{t}(z),
$$

and

$$
P_{z}(f)=\int_{0}^{\infty} H_{t}(f) N_{t}(z) d t / t
$$

Since $\int_{0}^{\infty} N_{t}(z) d t / t=1,\left\|P_{z}(f)\right\|_{p} \leqq\|f\|_{p}$ for $z>0$.

Proposition 1. $P_{z}$ is a strongly continuous contraction semigroup on $L^{p}(H)$. There is a Borel probability measure $p_{z}$ on $H$ such that $P_{z}(f)=\int_{H} T_{y} f d p_{z}(y)$.

Proof. The integral $P_{z}(f)=\int_{0}^{\infty} H_{t}(f) N_{t}(z) d t / t$ converges absolutely in $L^{p}(H)$ and $\left\|P_{z}(f)\right\|_{p} \leqq\|f\|_{p}$ as above.

$$
\left\|P_{z}(f)-f\right\|_{p} \leqq \int_{0}^{\infty}\left\|H_{t}(f)-f\right\|_{p} N_{t}(z) d t / t
$$

since $\int_{0}^{\infty} N_{t}(z) d t / t=1$. For $\varepsilon>0$ there is a $\delta>0$ such that $\left\|H_{t}(f)-f\right\|_{p}<\varepsilon$ when $0<t<\delta$. Thus

$$
\left\|P_{z}(f)-f\right\|_{p} \leqq \varepsilon+\int_{\delta}^{\infty}\left\|H_{t}(f)-f\right\|_{p} N_{t}(z) d t / t
$$

$N_{t}(z) / t \rightarrow 0$ as $z \downarrow 0$ and for $t \geqq \delta, N_{t}(z) / t \leqq K t^{-3 / 2}$. By the dominated convergence theorem, $\lim \sup _{z \downarrow 0}\left\|P_{z}(f)-f\right\|_{p} \leqq \varepsilon$ for every $\varepsilon>0$, and $\lim _{z \downarrow 0} P_{z}(f)=f$.

The set of Borel measurable functions $f$ on $H$ such that $f\left(t^{1 / 2} x\right)$ is Borel measurable on $(0, \infty) \times H$ is closed under pointwise limits and includes the continuous 
functions and hence consists of all Borel functions. Let $\chi_{E}(y)$ denote the characteristic function of a Borel set $E$ in $H$ and set $\mu_{t}(E)=\int_{H} \chi_{E}(y) d n_{t} \circ B^{-1}(y)$. Then $\mu_{t}(E)=\int_{H} \chi_{E}\left(t^{1 / 2} y\right) d n_{1} \circ B^{-1}(y)$ is a bounded measurable function of $t$. Set $p_{z}(E)$ $=\int_{0}^{\infty} \mu_{t}(E) N_{t}(z) d t / t$. By the bounded convergence theorem, $p_{z}(E)$ is a countably additive Borel measure on $H$. For simple functions, $F$, from $H$ to $L^{p}(H)$,

$$
\int_{H} \Gamma(y) d p_{z}(y)=\int_{0}^{\infty} \int_{H} F(y) d n_{t} \circ B^{-1}(y) N_{t}(z) d t / t .
$$

Since for each bounded continuous function $F$ from $H$ to $L^{p}(H)$, there is a bounded sequence of simple functions converging pointwise to $F(y)$, the above equality of integrals holds for all bounded continuous functions by the bounded convergence theorem. In particular, $P_{z}(f)=\int_{H} T_{y} f d p_{z}(y)$.

To show that $P_{z} \circ P_{w}=P_{z+w}$ we show that $p_{z} * p_{w}=p_{z+w}$ since $P_{z}\left(P_{w}(f)\right)=$ $\int_{H} T_{y} f d\left(p_{z} * p_{w}\right)(y)$. To prove this identity we shall calculate the Fourier transform, $\hat{p}_{z}$, of measure $p_{z}$. We shall see that $\hat{p}_{z}(y)=\exp \left[-z\left\|B^{*} y\right\|\right]$ and hence $\left(p_{z} * p_{w}\right)^{\wedge}(y)$ $=\exp \left[-(z+w)\left\|B^{*} y\right\|\right]$. Thus $p_{z} * p_{w}=p_{z+u}$, by uniqueness of the Fourier transforms of Borel measures [4].

It follows from the above considerations on the measure $p_{z}$ that

$$
\begin{aligned}
\hat{p}_{z}(u) & =\int_{0}^{\infty} \int_{H} \exp [i(u, y)] d n_{t} \circ B^{-1}(y) N_{t}(z) d t / t \\
& =\int_{0}^{\infty} \exp \left[-t / 4\left\|B^{*} u\right\|^{2}\right] N_{t}(z) d t / t .
\end{aligned}
$$

Set $a=2^{-1}\left\|B^{*} u\right\|$ and $F(a, z)=\int_{0}^{\infty} \exp \left[-t a^{2}\right] N_{t}(z) d t / t$ for $z>0$ and set $F(a, 0)=1$. $F(a, z)$ is continuous on $z \geqq 0$ for each $a \geqq 0$. If $s=t^{-1} z^{2}$,

$$
F(a, z)=\pi^{-1 / 2} \int_{0}^{\infty} \exp \left[-\frac{(z a)^{2}}{s}-s\right] d s / s^{1 / 2}
$$

and by a theorem on uniform convergence

$$
F_{2}(a, z)=\left(-2 z a^{2}\right) \pi^{-1 / 2} \int_{0}^{\infty} \exp \left[-\frac{(z a)^{2}}{s}-s\right] d s / s^{3 / 2}
$$

for $z>0$ and $a>0$. Set $t=s^{-1}(z a)^{2}$, to get $F_{2}(a, z)=-2 a F(a, z)$ and $F(a, z)=$ $F(a, 0) \exp [-2 a z]$. Thus $\hat{p}_{z}(u)=\exp \left[-z\left\|B^{*} u\right\|\right]$. This concludes the proof.

Remark. The operator $P_{z}$ is the Poisson integral of $f$. It follows from the fact that $\hat{p}_{z}(u)=\exp \left[-z\left\|B^{*} u\right\|\right]$ that if $H$ is $n$-dimensional Euclidean space and $B$ is the identity operator, then

$$
d p_{z}(y)=\frac{\Gamma((n+1) / 2)}{\pi^{(n+1) / 2}} z\left(\|y\|^{2}+z^{2}\right)^{-(n+1) / 2} d y,
$$

which is the Poisson kernel in $E_{n}$.

For $f$ in $L^{p}(H), T_{y} f$ is a bounded uniformly continuous function from $H$ to $L^{p}(H)$. If $B$ is a one-one Hilbert-Schmidt operator on $H, T_{B y} f$ is uniformly continuous in 
the norm $\|B x\|$ and $T_{B y} f$ extends uniquely to a continuous function from $H_{B}$, the completion of $H$ in the norm $\|B x\|$. In the next proposition we shall use the Wiener space representative for the normal distribution $n_{t}$, the normal distribution with variance parameter $t / 2$.

The Wiener space representative, $F$, of the normal distribution $n_{t}$ may depend on $t$ since for $y$ in $H^{*}, F(y)(s)$ is defined only almost everywhere. It is known [6], however, that $F$ may be chosen so that if $h_{n} \in H_{B}^{*}$ and $h_{n} \rightarrow h$ in $H^{*}$ then $F\left(h_{n}\right)$ converges to $F(h)$ in $L^{2}\left(H, n_{t}\right)$ for all $t>0$. We shall assume in what follows that we are working with this representative for the normal distribution $n_{t}$.

Denote by $y \rightarrow S_{y}$ the regular representation of the additive group of $H$ acting on $L^{1}\left(H, n_{t}\right)$. For $h$ in $H$, let $C_{h}$ denote the infinitesimal generator of the semigroup $S_{u h}, u>0$.

Proposition 2. Let $h_{1}, \ldots, h_{n}$ be in $H$, let $A_{h}^{\alpha}=A_{h_{1}}^{\alpha_{1}} \cdots A_{h_{n}}^{\alpha_{n}}$, and let $C_{h}^{\alpha}=C_{h_{1}}^{\alpha_{1}}$ $\cdots C_{h_{n}}^{\alpha_{n}}$. Then

and

$$
A_{h}^{\alpha} H_{t}(f)=\int_{H_{B}} T_{B y} f C_{h}^{\alpha}(1)(y) d n_{t}(y)
$$

$$
A_{h}^{\alpha} P_{z}(f)=\int_{0}^{\infty} A_{h}^{\alpha} H_{t}(f) N_{t}(z) d t / t
$$

$P_{z}(f)$ is an infinitely differentiable function of $z$ for $z>0$ and

$$
\frac{d^{n}}{d z^{n}} P_{z}(f)=\int_{0}^{\infty} H_{t}(f) \frac{d^{n}}{d z^{n}} N_{t}(z) d t / t
$$

Proof. For $t>0$ and $h$ in $H$,

$$
\begin{aligned}
A_{h} H_{t}(f) & =\lim _{s \downarrow 0} s^{-1}\left(T_{s B h} H_{t}(f)-H_{t}(f)\right) \\
& =\lim _{s \downarrow 0} H_{t}\left(s^{-1}\left(T_{s B h} f-f\right)\right) \\
& =\lim _{s \downarrow 0} s^{-1} \int_{H}\left(T_{B(y+s h)} f-T_{B y} f\right) d n_{t}(y) .
\end{aligned}
$$

Let $g \in L^{p^{\prime}}(H), p^{\prime}=p /(p-1)$ and $\langle$,$\rangle denote the dual pairing between L^{p}$ and $L^{p^{\prime}}$. Set $\phi(y)=\left\langle T_{B y} f, g\right\rangle$. By Theorem 3 of [9],

where

$$
\int_{H_{B}} \phi(y+s h) d n_{t}(y)=\int_{H_{B}} \phi(y) D_{t / 2}(y, s h) d n_{i}(y)
$$

Thus

$$
D_{p}(x, y)=\exp \left[\frac{(x, y)}{p}-\frac{\|y\|^{2}}{2 p}\right]
$$

$$
\begin{gathered}
A_{h} H_{t}(f)=\lim _{s \downarrow 0} s^{-1} \int_{H_{B}} T_{B y} f\left[D_{t / 2}(y, s h)-1\right] d n_{t}(y) \cdot s^{-1}\left[D_{l / 2}(y-s h)-1\right] . \\
s^{-1}\left[D_{t / 2}(y, s h)-1\right]=s^{-1}\left[S_{s h}(1)(y)-1\right]
\end{gathered}
$$


converges to $C_{h}(1)$ in $L^{1}\left(H, n_{t}\right)$ as $s \downarrow 0$ and

$$
\left|s^{-1}\left(S_{s h}(1)(y)-1\right)\right| \leqq \text { const. } \exp [2 / t|(h, y)|](|(h, y)|+1)
$$

for $s \leqq 1$, so that by the dominated convergence theorem,

$$
A_{h} H_{t}(f)=\int_{H_{B}} T_{B y} f C_{h}(1)(y) d n_{t}(y) .
$$

Proceed by induction. Let $\alpha=\left(\alpha_{1}, \ldots, \alpha_{i}, \ldots, \alpha_{n}\right)$ and $\beta=\left(\alpha_{1}, \ldots, \alpha_{i}+1, \ldots, \alpha_{n}\right)$.

$$
A_{h}^{\beta} H_{t}(f)=\lim _{s \downarrow 0} s^{-1} \int_{H_{B}}\left(T_{B\left(y+s h_{i}\right)} f-T_{B y} f\right) C_{h}(1)(y) d n_{t}(y) .
$$

Let $g \in L^{p^{\prime}}(H)$ and set $\phi(y)=\left\langle T_{B y} f, g\right\rangle$.

$$
\begin{aligned}
\left\langle s^{-1} \int_{H_{B}}\right. & \left.\left(T_{B\left(y+s h_{t}\right)} f-T_{B y} f\right) C_{h}^{\alpha}(1)(y) d n_{t}(y), g\right\rangle \\
& =s^{-1} \int_{H_{B}}\left(\phi\left(y+s h_{i}\right)-\phi(y)\right) C_{h}^{\alpha}(1)(y) d n_{t}(y) .
\end{aligned}
$$

If $\phi$ were a bounded tame function, this last integral would be

$$
\int_{H_{B}} \phi(y) s^{-1}\left[S_{s h_{i}} C_{h}^{\alpha}(1)(y)-C_{h}^{\alpha}(1)(y)\right] d n_{t}(y) .
$$

Since the bounded tame functions are weakly dense in $L^{\infty}\left(H, n_{t}\right)$, this equality holds for all bounded $n_{t}$-measurable functions $\phi$. Thus

$$
A_{h}^{\beta} H_{t}(f)=\lim _{s \downarrow 0} s^{-1} \int_{H_{B}} T_{B y} f\left[S_{s h_{i}} C_{h}^{\alpha}(1)(y)-C_{h}^{\alpha}(1)(y)\right] d n_{t}(y) .
$$

Each $C_{h}^{\alpha}(1)(y)$ is a polynomial and $s^{-1}\left[S_{s h_{t}} C_{h}^{\alpha}(1)(y)-C_{h}^{\alpha}(1)(y)\right]$, the difference quotient, is dominated in absolute value by an $n_{t}$-integrable tame function which is independent of $s$ for sufficiently small $s$. Thus the dominated convergence theorem assures that $A_{h}^{\beta} H_{t}(f)$ exists in $L^{p}(H)$ and that the desired equation holds.

Similarly one shows that the difference quotients $s^{-1}\left(T_{s B h_{i}} A_{h}^{\alpha} H_{t}(f)-A_{h}^{\alpha} H_{t}(f)\right)$ are dominated in $p$-norm by a polynomial in $t^{-1 / 2}$. Hence the differentiability of $P_{z}(f)$ follows from the dominated convergence theorem; $A_{h}^{\alpha} P_{z}(f)$ has the desired form.

Each of the difference quotients $h^{-1}\left[N_{t}^{(k)}(z+h)-N_{t}^{(k)}(z)\right]$ is dominated in absolute value by a function of the form $p\left(z, t^{-1 / 2}\right) \exp \left[-z^{2} / t\right]$ where $p\left(z, t^{-1 / 2}\right)$ is a polynomial in each variable which is independent of $h$ for sufficiently small $h$. Differentiability of $P_{z}(f)$ in $z$ now follows from the dominated convergence theorem.

We now turn to the characterization of the bounded harmonic functions $U:(0, \infty) \rightarrow L^{2}(H)$. Let $p(x)$ be a polynomial on $H$. Set

$$
W(p)(y)=\int_{H} p\left(2^{1 / 2} x+i y\right) d n(x) .
$$


$W(p)$ is the Wiener transform of $p(x)$ and $W$ extends to a unitary operator on $L^{2}(H)$ (see [8]). If $f \in L^{2}(H), W\left(T_{y} f\right)=\exp [i F(y) / 2] W(f)$ where $F$ is the representative of the normal distribution with variance parameter 1 . Hence if $f \in L^{2}(H)$ is in the domain of $A_{j}, W\left(A_{j} f\right)=\left[i F\left(B e_{j}\right) / 2\right] W(f)$. Thus if $U:(0, \infty) \rightarrow L^{2}(H)$ is harmonic; i.e. $U_{z z}(z)+\sum_{j=1}^{\infty} A_{j}^{2} U(z)=0$ for $z>0$, then

$$
W(U(z))_{z z}-\frac{\left(\left\|B^{*}(\cdot)\right\|^{\sim}\right)^{2}}{4} W(U(z))=0
$$

where $\left\|B^{*}(\cdot)\right\| \sim$ is the measurable function determined by $\left\|B^{*} u\right\|$. In particular if $f \in L^{2}(H)$ the Poisson integral, $P_{z}(f)$, of $f$ is harmonic. For as was shown in the proof of Proposition 1, the Fourier transform, $\hat{p}_{z}$, of the measure $p_{z}$ is $\hat{p}_{z}(u)$ $=\exp \left[-z\left\|B^{*} u\right\|\right]$. Since $W\left(P_{z}(f)\right)=W(f) \int_{H} \exp [i F(y) / 2] d p_{z}(y)$, we have that $W\left(P_{z}(f)\right)=W(f) \exp \left[-z / 2\left\|B^{*}(\cdot)\right\| \sim\right]$. Hence

$$
W\left(P_{z}(f)\right)_{z z}=\frac{\left(\left\|B^{*}(\cdot)\right\|^{2}\right)^{2}}{4} W\left(P_{z}(f)\right)
$$

and $P_{z}(f)$ is harmonic.

Theorem 1. If $f$ is in $L^{2}(H)$ and if $P_{z}(f)$ is its Poisson integral, $P_{z}(f)$ is harmonic and is bounded as a function of $z, z>0$, to $L^{2}(H)$. Conversely, if $U:(0, \infty) \rightarrow L^{2}(H)$ is a harmonic function such that $\|U(z)\|_{2}$ is a bounded function of $z$, there is a unique function $f$ in $L^{2}(H)$ such that $U(z)=P_{z}(f)$.

Proof. The facts that $P_{z}(f)$ is bounded in $z$ and is harmonic were established above.

Let $\|U(z)\|_{2} \leqq C<\infty$ for $z>0$ and let $U$ be harmonic. Let $z_{1}, z_{2}, z_{3}, \ldots$ be a sequence of positive real numbers which converges to zero. By virtue of the boundedness of the sequence $\left\{U\left(z_{i}\right)\right\}$ in $L^{2}(H)$ and by virtue of the weak compactness of a closed ball in $L^{2}(H)$ there is a subsequence, again denoted by $\left\{U\left(z_{i}\right)\right\}$, and a function $f$ in $L^{2}(H)$ such that $\left\{U\left(z_{i}\right)\right\}$ converges weakly to $f$.

Set $\phi(z)=U\left(z+z_{i}\right)-P_{z}\left(U\left(z_{i}\right)\right)$. Then $\phi(0)=0$ and

$$
\begin{aligned}
W(\phi(z))_{z z} & =W\left(U\left(z+z_{i}\right)\right)_{z z}-W\left(P_{z}\left(U\left(z_{i}\right)\right)\right)_{z z} \\
& =\frac{\left(\left\|B^{*}(\cdot)\right\| \sim\right)^{2}}{4}\left[W\left(U\left(z+z_{i}\right)\right)-W\left(P_{z}\left(U\left(z_{i}\right)\right)\right)\right] \\
& =\frac{\left(\left\|B^{*}(\cdot)\right\| \sim\right)^{2}}{4} W(\phi(z)) .
\end{aligned}
$$

Set $a=2^{-1}\left\|B^{*}(\cdot)\right\|^{\sim}$ and $\psi(z)=W(\phi(z))$. We have $\psi^{\prime \prime}(z)=a^{2} \psi(z)$ or $\psi(z)=A \exp (a z)$ $+B \exp (-a z)$. Since $\psi(0)=0, B=-A$ and $\psi(z)=2 A \operatorname{Sinh}(a z) ; A$ is a measurable function. Since $a>0$ and $z>0,|2 A \operatorname{Sinh}(a z)| \geqq 2 a z|A|$. Since $\|U(z)\|_{2} \leqq C,\|\psi(z)\|_{2}$ $=\|\phi(z)\|_{2} \leqq 2 C$; but $z\|A\| B^{*}(\cdot)\|\sim\|_{2} \leqq\|\psi(z)\|_{2}$ and the left side of this inequality is unbounded in $z$ unless $A=0$ almost everywhere. Hence $\phi(z)=0$ for $z \geqq 0$ and $U\left(z+z_{i}\right)=P_{z}\left(U\left(z_{i}\right)\right)$. 
If $g$ is in $L^{2}(H)$,

$\left\langle g, P_{z}\left(U\left(z_{i}\right)\right)\right\rangle=E\left(g P_{z}\left(U\left(z_{i}\right)\right)\right)=\int_{H}\left\langle g, T_{y} U\left(z_{i}\right)\right\rangle d p_{z}(y)=\int_{H}\left\langle T_{y}^{*} g, U\left(z_{i}\right)\right\rangle d p_{z}(y)$.

Since $\left\{U\left(z_{i}\right)\right\}$ converges weakly to $f$ as $z_{i}$ tends to zero, $\left\langle g, P_{z}\left(U\left(z_{i}\right)\right)\right\rangle$ converges to $\left\langle g, P_{z}(f)\right\rangle$ as $z_{i}$ tends to zero by the bounded convergence theorem. Since $U\left(z+z_{i}\right)$ $=P_{z}\left(U\left(z_{i}\right)\right),\langle g, U(z)\rangle=\left\langle g, P_{z}(f)\right\rangle$ for all $g$ in $L^{2}(H)$. Hence $U(z)=P_{z}(f)$. The uniqueness of $f$ follows by taking Wiener transforms and noting that the Fourier transform of $p_{z}$ does not vanish.

REMARK 1. Let $1<p<\infty$ and $f \in L^{p}(H)$ satisfy $\left\|T_{y} f-f\right\|_{p} \leqq K\|y\|$ where $K$ is a finite positive constant and $y \rightarrow T_{y}$ is the regular representation of $H$ on $L^{p}(H)$. We shall show that $P_{z}(f)$ is harmonic in a weak sense. Let $g \in L^{p^{\prime}}(H), p^{\prime}=p /(p-1)$. Then $\phi(y)=\left\langle g, T_{B y} f\right\rangle$ is a Lip 1 function on $H_{B}$. In the notation of [6] $p_{t} \phi(x)$ $=\left\langle g, T_{B x} H_{t}(f)\right\rangle=v(t, x)$. By Theorem 3 of [6], $v(t)=v(t, 0)$ is a boundedly and continuously differentiable function of $t$ and

$$
d v / d t=\left.\frac{1}{4} \operatorname{trace} D^{2} v(t, x)\right|_{x=0}
$$

where $D^{2}$ is the second Fréchet derivative of $v$ with respect to the second variable. (The change in the above constant is due to the fact that we have replaced Gross' parameter $t$ by $t / 2$.) $\int_{0}^{\infty}(d v / d t) N_{t}(z) d t / t$ exists and integration by parts shows that this integral is $-\frac{1}{4}\left\langle g, P_{z}(f)_{z z}\right\rangle$. By formula 9, p. 44 of [6],

$$
\left(\left(D^{2} p_{t} \phi\right)(x) h_{1}, h_{2}\right)=2 / t \int_{H_{B}} \phi(x+y)\left\{2 / t F\left(h_{1}\right)(y) F\left(h_{2}\right)(y)-\left(h_{1}, h_{2}\right)\right\} d n_{t}(y) .
$$

Thus

$$
\left.\operatorname{trace} D^{2} v(t, x)\right|_{x=0}=\sum_{j=1}^{\infty} 2 / t \int_{H_{B}} \phi(y)\left\{2 / t F\left(e_{j}\right)(y)^{2}-1\right\} d n_{t}(y)=\sum_{j=1}^{\infty}\left\langle g, A_{j}^{2} H_{t}(f)\right\rangle
$$

by Proposition 2. By inequality 38, p. 173 of [6], the trace class norm of $D^{2} v(t, 0)$ is dominated by $K t^{-1 / 2}$ where $K$ is a positive constant. Hence $\sum_{j=1}^{n}\left|\left\langle g, A_{j}^{2} H_{t}(f)\right\rangle\right|$ is dominated by $K t^{-1 / 2}$ for all $n$. By the dominated convergence theorem we get that $P_{z}(f)_{z z}+\sum_{j=1}^{\infty} A_{j}^{2} P_{z}(f)$ converges weakly to zero.

REMARK 2. An argument similar to that given in the proof of Theorem 1 shows that the bounded differentiable functions $V:(0, \infty) \rightarrow L^{2}(H)$ which satisfy $V^{\prime}(z)$ $=\sum_{j=1}^{\infty} A_{j}^{2} V(z)$ for $z>0$ must be of the form $V(t)=H_{t}(f)$ for some unique $f$ in $L^{2}(H) . H_{t}$ is as in equation (2).

3. Conjugate harmonic functions. Let $B$ be the one-one Hilbert-Schmidt operator of $\S 2$ and let $e_{1}, e_{2}, e_{3}, \ldots$ be the orthonormal basis for the Hilbert space $H$. Let $n_{t}(z)=(\pi t)^{-1 / 2} \exp \left[-z^{2} / t\right]$ and let the representative of the normal distribution be the map $F$ from $H^{*}$ to Borel measurable functions on $H_{B}$; choose $F$ so that it is independent of the variance parameter. For $f$ in $L^{p}(H), 1<p<\infty$, set

$$
Q_{z}^{(j)}(f)=\lim _{R \rightarrow \infty} \int_{0}^{R}\left[\int_{H_{B}} T_{B y} f F\left(e_{j}\right)(y) d n_{t}(y)\right] n_{t}(z) d t / t .
$$


Proposition 3. $Q_{z}^{(j)}$ is a bounded operator on $L^{p}(H)$ for $1<p<\infty$.

Proof. Let $e$ denote a generic element of the o.n. basis of $H$ and let $Q_{z}$ denote the conjugate Poisson integral corresponding to $e$.

$$
\begin{aligned}
\int_{0}^{R}\left[\int_{H_{B}} T_{B y} f F(e)(y) d n_{t}(y)\right] n_{t}(z) d t / t \\
=\pi^{-1 / 2} \int_{0}^{R}\left[\int_{H_{B}} T_{t^{1 / 2} B y} f F(e)(y) d n_{1}(y)\right] \exp \left[\frac{-z^{2}}{t}\right] d t / t ;
\end{aligned}
$$

for let $\phi(y)=\left\langle g, T_{B y} f\right\rangle$ with $g$ in $L^{p^{\prime}}(H), p^{\prime}=p /(p-1)$. If $\phi(y)$ were a bounded tame function,

$$
\int_{H_{B}} \phi(y) F(e)(y) d n_{t}(y)=t^{1 / 2} \int_{H_{B}} \phi\left(t^{1 / 2} y\right) F(e)(y) d n_{1}(y) .
$$

Since the bounded tame functions are weakly dense in $L^{\infty}(H)$, the desired equality holds for $\phi(y)=\left\langle g, T_{B y} f\right\rangle$ and the first assertion holds.

Set $s^{2}=t$ so that the above integral is

$$
2 \pi^{-1 / 2} \int_{0}^{\rho}\left[\int_{H_{B}} T_{s B u .} f F(e)(y) d n_{1}(y)\right] \exp \left[\frac{-z^{2}}{s^{2}}\right] d s / s, \quad \text { where } \rho=R^{1 / 2}
$$

Since $F(e)(y)$ is an odd function, this integral is

$$
\int_{-\infty}^{0}\left[\int_{H_{B}} T_{. s B y .} f F(e)(y) d n_{1}(y)\right] \exp \left[\frac{-z^{2}}{s^{2}}\right] d s / s .
$$

Thus

$$
Q_{\approx}(f)=\pi^{-1: 2} \lim _{R \rightarrow \infty} \int_{|t| \leqq R}\left[\int_{H_{B}} T_{t B y} f F(e)(y) d n_{1}(y)\right] \exp \left[\frac{-z^{2}}{t^{2}}\right] d t / t .
$$

Let

$$
Q_{z}^{R}(f)=\pi^{-1 / 2} \int_{|t| \leqq R}\left[\int_{H_{B}} T_{t B y} f F(e)(y) d n_{1}(y)\right] \exp \left[\frac{-z^{2}}{t^{2}}\right] d t / t
$$

Then

$$
Q_{z}^{R}(f)=\pi^{-1 / 2} \int_{H_{B}}\left[\int_{|t| \leqq R} T_{t B y} \exp \left[\frac{-z^{2}}{t^{2}}\right] d t / t\right] F(e)(y) d n_{1}(y) .
$$

By Minkowski’s integral inequality,

$$
\left\|Q_{z}^{R}(f)\right\|_{p} \leqq \int_{H_{B}}\left\|\int_{|t| \leqq R} T_{t B y} f \exp \left[\frac{-z^{2}}{t^{2}}\right] d t / t\right\|_{p}|F(e)(y)| d n_{1}(y) .
$$

Set $s=t\|B y\|$ so that

$$
\begin{aligned}
& \int_{|t| \leqq R} T_{t B y} f \exp \left[\frac{-z^{2}}{t^{2}}\right] d t / t \\
& \quad=\int_{|t| \leqq R\|B y\|} T_{t \omega} f \exp \left[\frac{-z^{2}\|B y\|^{2}}{t^{2}}\right] d t / t \quad \text { where } \omega=B y\|B y\|^{-1} .
\end{aligned}
$$


Let $f$ be a bounded continuous tame function. Since the normal distribution on $H$ is orthogonally invariant,

$$
\begin{aligned}
& \begin{aligned}
\left\|\int_{|t| \leqq R\|B y\|} T_{t \omega} f \exp \left[\frac{-z^{2}\|B y\|^{2}}{t^{2}}\right] d t / t\right\|_{p}^{p} \\
=\int_{-\infty}^{\infty} \cdots \int_{-\infty}^{\infty} \mid \int_{|s| \leqq R\|B y\|} f\left(x_{1}-s, x_{2}, \ldots, x_{n}\right) \exp \left[\frac{x_{1} s}{p}-\frac{s^{2}}{2 p}\right] \\
\left(^{*}\right) \quad \times \exp \left[\frac{-z^{2}\|B y\|^{2}}{s^{2}}\right] d s /\left.s\right|^{p} d n\left(x_{1}\right) \cdots d n\left(x_{n}\right)
\end{aligned} \\
& =(2 \pi)^{-1 / 2} \int_{-\infty}^{\infty} \cdots \int_{-\infty}^{\infty} \mid \int_{|s| \leqq R\|B y\|} f\left(x_{1}-s, x_{2}, \ldots, x_{n}\right) \exp \left[\frac{-\left(x_{1}-s\right)^{2}}{2 p}\right] \\
& \times \exp \left[\frac{-z^{2}\|B y\|^{2}}{s^{2}}\right] d s /\left.s\right|^{p} d x_{1} d n\left(x_{2}\right) \cdots d n\left(x_{n}\right) .
\end{aligned}
$$

Hence to prove that the $\left\|Q_{z}^{R}(f)\right\|_{p}$ are uniformly bounded, it suffices to show that

$$
T_{z}^{r}(f)=\int_{|t| \leqq r} f(x-t) \exp \left[\frac{-z^{2}}{t^{2}}\right] d t / t
$$

is a uniformly bounded family of operators on $L^{p}((-\infty, \infty), d x), 1<p<\infty$. Let $f$ be a smooth rapidly decreasing function. Then the Fourier transform $\hat{T}_{z}^{r}(f)(u)$ of $T_{z}^{\gamma}(f)$ is

$$
2 i \hat{f}(u) \int_{0}^{r} \sin (u y) \exp \left[\frac{-z^{2}}{y^{2}}\right] d y / y \text {. }
$$

Let

$$
\phi_{r}(u)=\int_{0}^{r} \sin (u y) \exp \left[\frac{-z^{2}}{y^{2}}\right] d y / y .
$$

Then $\phi_{r}(u)=\int_{0}^{r u} \sin y \exp \left[-u^{2} z^{2} / y^{2}\right] d y / y$ for $u \neq 0$ and $\phi_{r}(0)=0$. Now,

$$
\rho \int_{\rho / 2}^{\rho}\left|\phi_{r}^{\prime}(u)\right|^{2} d u
$$

can be estimated as follows.

$$
\phi_{r}^{\prime}(u)=\int_{0}^{r u} \exp \left[\frac{-u^{2} z^{2}}{y^{2}}\right] \sin y\left[\frac{-2 u z^{2}}{y^{2}}\right] d y / y+\exp \left[\frac{-z^{2}}{r^{2}}\right] \sin (u r) / u
$$

and

$$
\left(\int_{\rho / 2}^{\rho}\left|\phi_{r}^{\prime}(u)\right|^{2} d u\right)^{1 / 2} \leqq K \int_{0}^{\infty} \exp \left[\frac{-\rho^{2} z^{2}}{4 y^{2}}\right] \frac{z^{2}}{y^{2}}\left(\int_{\rho / 2}^{\rho} u^{2} d u\right)^{1 / 2} d y / y+K \rho^{-1 / 2} \leqq K_{1} \rho^{-1 / 2}
$$

Thus $\rho \int_{\rho / 2}^{o}\left|\phi_{r}^{\prime}(u)\right|^{2} d u \leqq K_{1}<\infty$. By Hörmander's Theorem on $L^{p}$-multipliers [7], the $T_{z}^{r}$ form a uniformly bounded family of operators on $L^{p}((-\infty, \infty), d x)$. Hence the $Q_{z}^{R}$ form a bounded family on $L^{p}(H)$. 
We shall prove that the $Q_{z}^{R}$ converge strongly as $R$ tends to infinity. It is sufficient to show that the $T_{z}^{r}$ converge strongly as $r \rightarrow \infty$. Then two applications of the dominated convergence theorem show that the $Q_{z}^{R}$ converge strongly.

$$
T_{z}^{r}(f)-T_{z}^{o}(f)= \pm \int_{\rho \leqq|y| \leqq r} f(x-y) \exp \left[\frac{-z^{2}}{y^{2}}\right] d y / y .
$$

Let $f$ be continuous with compact support and let $\alpha, \beta>1$ satisfy $1 / p=1 / \alpha+1 / \beta-1$. Apply Young's inequality to the above difference to get

$$
\left\|T_{z}^{r}(f)-T_{z}^{\rho}(f)\right\|_{p} \leqq\|f\|_{\alpha}\left(\int_{\rho \leqq|y| \leqq r} y^{-\beta} d y\right)^{1 / \beta}
$$

The right side of the last inequality tends to zero as $\rho, r$ tend to infinity. A density argument completes the proof of the strong convergence of the $T_{z}^{r}$.

For bounded continuous tame functions $f$ on $H$

$$
\left\|\int_{|t| \leqq r} T_{t B y} f \exp \left[\frac{-z^{2}}{t^{2}}\right] d t / t\right\|_{p} \leqq K\|f\|_{p}
$$

where $K$ is a constant which is independent of $z, y$, and $r$. Hence by the dominated convergence theorem,

$$
\lim _{R, \rho \rightarrow \infty}\left\|Q_{z}^{R}(f)-Q_{z}^{\rho}(f)\right\|_{p} \leqq \int_{H_{B}} \lim _{R, \rho \rightarrow \infty}\left\|\int_{\rho \leqq|t| \leqq R} T_{t B y} f \exp \left[\frac{-z^{2}}{t^{2}}\right] d t / t\right\|_{p}|F(e)(y)| d n_{1}(y) .
$$

Use the fact that $f$ is tame to write $\left\|\int_{o \leqq|t| \leqq R} T_{t B y} f \exp \left[-z^{2} / t^{2}\right] d t / t\right\|_{p}$ as in $\left(^{*}\right)$. Since the $T_{z}^{\tau}(f)$ are uniformly bounded, we may apply the dominated convergence theorem to conclude that the $Q_{z}^{R}(f)$ converge in $L^{p}(H)$. A density argument completes the proof.

For $f$ in $L^{p}(H), 1<p<\infty$, the $Q_{z}^{(j)}(f)$ are the conjugate Poisson integrals of $f$.

If one applies the Wiener transform to the operator $Q_{z}^{(j)}$ on $L^{2}(H)$ one sees that

$$
W\left(Q_{z}^{(j)}(f)\right)=i \frac{\left(B^{*} e_{j}, \cdot\right)^{\sim}}{\left\|B^{*}(\cdot)\right\|^{\sim}} \exp \left[-z / 2\left\|B^{*}(\cdot)\right\|^{\sim}\right] W(f)
$$

Hence, at least in $L^{2}(H), Q_{z}^{(j)}=P_{z} \circ R_{j}$ where $R_{j}$ is the singular integral operator

$$
R_{j}(f)=\lim _{\rho \rightarrow \infty ; \delta \downarrow 0} \int_{\delta}^{\rho}\left[\int_{H_{B}} T_{t B y} f F\left(e_{j}\right)(y) d n(y)\right] d t / t
$$

These operators have been studied in [1]

Proposition 4. For $1<p<\infty, Q_{z}^{(j)}=P_{z} \circ R_{j}$ as an operator on $L^{p}(H)$.

Proof. Let $e$ denote a generic element of the basis $e_{1}, e_{2}, e_{3}, \ldots$ of $H$ and let $Q$ and $R$ be the corresponding operators. Let $F$ be the usual representative of the 
normal distribution which maps to Borel measurable functions on the Wiener space $H_{B}$. Let $S$ be a finite dimensional orthogonal projection on $H$. Set

$$
\begin{aligned}
& Q_{z}^{S}(f)=\lim _{R \rightarrow \infty} \int_{0}^{R}\left[\int_{H_{B}} T_{S B y} f F(e)(y) d n_{t}(y)\right] n_{t}(z) d t / t, \\
& P_{z}^{S}(f)=\int_{0}^{\infty}\left[\int_{H} T_{S y} f d n_{t} \circ B^{-1}(y)\right] N_{t}(z) d t / t,
\end{aligned}
$$

and

$$
R^{S}(f)=\lim _{\delta \rightarrow 0 ; \rho \rightarrow \infty} \int_{\delta}^{o}\left[\int_{H_{B}} T_{t S B y} f F(e)(y) d n(y)\right] d t / t
$$

We may suppose that $f \geqq 0$. Each of these operators is bounded independently of $\mathrm{S}$ on $L^{p}(H)$.

Let $Q_{z}^{S \rho}(f)=\int_{0}^{o}\left[\int_{H_{B}} T_{S B y} f F(e)(y) d n_{t}(y)\right] n_{t}(z) d t / t$. Let $f$ be a bounded continuous tame function on $H$ and let $V$ be the orthogonal projection onto the span of $e$ and the base of $f$. Let $S>V$. We shall show that $Q_{z}^{S o}(f)$ converges uniformly in $S$ to $Q_{z}^{S}(f)$ as $\rho \rightarrow \infty$.

$$
\begin{aligned}
\left\|Q_{z}^{S \rho}(f)-Q_{z}^{S t}(f)\right\|_{p} & \leqq K\left\|\int_{r}^{\rho}\left[\int_{H_{B}} T_{t^{1 / 2} S B y} f F(e)(y) d n_{1}(y)\right] \exp \left[\frac{-z^{2}}{t}\right] d t / t\right\|_{p} \\
& \leqq K \int_{H_{B}}\left\|\int_{r}^{\rho} T_{t^{1 / 2} S B y} f \exp \left[\frac{-z^{2}}{t}\right] d t / t\right\|_{p}|F(e)(y)| d n_{1}(y)
\end{aligned}
$$

by Fubini's theorem and Minkowski's inequality. Since $S>V$ and $f$ is based in $V H$, $T_{t^{1 / 2} S B y} f=\left(T_{t^{1 / 2} V B y} f\right) D_{p}\left(\cdot, t^{1 / 2}(S-V) B y\right)$. For each $y$ in $H_{B}$ the functions on the right are independent tame functions. The first is based in $V H$ and the second is based in $(S-V) H$. The product is based in $S H$. We write the normal distribution on $\mathrm{SH}$ as a product and apply Minkowski's inequality to get

$$
\left\|\int_{r}^{\rho} T_{t^{1 / 2} S_{B y}} f \exp \left[\frac{-z^{2}}{t}\right] d t / t\right\|_{p} \leqq \| \int_{r}^{\rho} T_{t^{1 / 2}{ }_{V B y}} f \exp \left[\frac{-z^{2}}{t}\right] d t /\left.t\right|_{p} .
$$

Set $u^{2}=t$ and $\omega=V B y\|V B y\|^{-1}$, use the fact that $f$ is tame and based on $V H$ and the fact that the normal distribution is rotationally invariant. Let $1 / p=1 / \alpha+1 / \beta-1$, $\alpha, \beta>1$ and apply Young's inequality. It follows that

$$
\left\|Q_{z}^{S \rho}(f)-Q_{z}^{S R}(f)\right\|_{p} \leqq K \delta(\rho, R)\left(\int_{H_{B}}\left\|V B y^{\prime}\right\|^{(1-\alpha) ! \alpha}|F(e)(y)| d n_{1}(y)\right)
$$

where $\delta(\rho, R)$ tends to zero as $\rho, R \rightarrow \infty$ and where $K$ is independent of $S$. We may choose $\alpha$ so that $\alpha<2$. By Hölder's inequality the last integral is dominated by $K\left(\int_{H}\|V B y\|^{1-\alpha} d n_{1}(y)\right)^{1 / 2}$. Let $K_{1}$ denote the kernel of $V B$. On $K_{1}^{\perp}, V B$ is a one-one finite dimensional operator into $H$. So there is a constant $C$ such that $\|y\| \leqq C\|V B y\|$ 
for $y$ in $K_{1}$. Write the normal distribution on $K_{1}^{\perp}$ in polar coordinates; if $V$ is sufficiently large, $\operatorname{dim}\left(K_{1}\right) \geqq 2$, the last integral is finite. Thus as $r, \rho \rightarrow \infty$,

$$
\left\|Q_{z}^{S o}(f)-Q_{z}^{S r}(f)\right\|_{p}
$$

converges to zero uniformly in $S$ when $f$ is a tame function and $S$ is sufficiently large.

Thus if $f$ is a bounded continuous tame function,

$$
\left\|Q_{z}(f)-Q_{z}^{S}(f)\right\|_{p} \leqq\left\|Q_{z}(f)-Q_{z}^{\rho}(f)\right\|_{p}+\left\|Q_{z}^{\rho}(f)-Q_{z}^{S o}(f)\right\|_{p}+\left\|Q_{z}^{S \rho}(f)-Q_{z}^{S}(f)\right\|_{p} \text {. }
$$

For $\varepsilon>0$, there is a $\rho_{0}$ such that the first and third terms on the right are each $<\varepsilon / 3$ when $\rho \geqq \rho_{0}$. Fix $\rho \geqq \rho_{0}$. By the strong continuity of the regular representation of $H$ acting on $L^{p}(H)$ and by the bounded convergence theorem, the second term on the right converges to zero as $S$ tends strongly to the identity through the directed set of finite dimensional projections on $H$. Hence $\lim _{S \rightarrow I} Q_{z}^{S}(f)=Q_{z}(f)$. Since the bounded continuous tame functions are dense in $L^{p}(H), Q_{z}$ is the strong limit of the net $Q_{z}^{S}$.

A similar argument shows that $R$ is the strong limit of the net $R^{S}$ [2]. The dominated convergence theorem implies that $P_{z}$ is the strong limit of the $P_{z}^{S}$.

Let $f$ be a bounded continuous tame function. Then $Q_{z}^{S}(f)$ and $P_{z}^{S}\left(R^{S}(f)\right)$ are tame functions. By taking Wiener transforms (with variance parameter $p / 2$ ) of each of these functions we see that $Q_{z}^{S}(f)=P_{z}^{S}\left(R^{S}(f)\right)$. Since the $Q_{z}^{S}, P_{z}^{S}$, and $R^{S}$ are uniformly bounded in $S$, we may take the limit in $S$ on each side of this equality. This gives the conclusion of the proposition.

In what follows we shall consider only the case $p=2$. For $f$ in $L^{2}(H)$ we set $U(z)=P_{z}(f), v_{j}(z)=-Q_{z}^{(j)}(f), V(z)=\left(v_{1}, v_{2}, v_{3}, \ldots\right)$, and $F(z)=(U(z), V(z))$.

Proposition 5. $\left(\partial_{l} / \partial z\right) U(z)+\sum_{j=1}^{\infty} A_{j} v_{j}(z)$ converges to zero in $L^{2}(H)$. For $i, j=1,2,3, \ldots A_{j} v_{i}=A_{i} v_{j}$, and $A_{j} U=(\partial / \partial z) v_{j}(z)$.

Proof. We shall first calculate the Wiener transform of $v_{j}$. By Proposition 4, $v_{j}(z)=-P_{z}\left(R_{j}(f)\right)$. From $\S 2$ we know that $W\left(P_{z}(f)\right)=W(f) \exp \left[-z / 2\left\|B^{*}(\cdot)\right\|\right]$. From [1] we have that $W\left(R_{j}(g)\right)=W(g)\left[i\left(B^{*}(\cdot), e_{j}\right)\left\|B^{*}(\cdot)\right\|^{-1}\right]$. Thus $(\partial / \partial z) U(z)$ $+\sum_{j=1}^{N} A_{j} v_{j}(z)$ has Wiener transform

$$
-\frac{1}{2}\left[\left\|B^{*}(\cdot)\right\|-\left\|B^{*}(\cdot)\right\|^{-1}\left(\sum_{j=1}^{N}\left(B^{*}(\cdot), e_{j}\right)^{2}\right)\right] \exp \left[-z / 2\left\|B^{*}(\cdot)\right\|\right] W(f) .
$$

The first term in this product converges to zero in measure. For each $N$, this quantity is dominated in absolute value by a constant multiple of $|W(f)|$. By the dominated convergence theorem $(\partial / \partial z) U(z)+\sum_{j=1}^{\infty} A_{j} v_{j}(z)$ converges to zero in $L^{2}(H)$.

The other assertions of the proposition follow by taking Wiener transforms.

The vector $F(z)=(U(z), V(z))$ has $|F(z)|=\left(U(z)^{2}+\sum_{j=1}^{\infty} v_{j}(z)^{2}\right)^{1 / 2}$ in $L^{2}(H)$ for each $z$ and $\||F(z)|\|_{2}=2^{1 / 2}\|U(z)\|_{2}$. For

$$
E\left(\sum_{j=m}^{n} v_{j}(z)^{2}\right)=E\left(|W(U)|^{2} \sum_{j=m}^{n}\left(B^{*}(\cdot), e_{j}\right)^{2}\left\|B^{*}(\cdot)\right\|^{-2}\right)
$$


$\sum_{j=m}^{n}\left\|B^{*}(\cdot)\right\|^{-2}\left(B^{*}(\cdot), e_{j}\right)^{2}$ converges boundedly to zero in measure as $n, m \rightarrow \infty$. The dominated convergence theorem implies that $E\left(\sum_{j=m}^{n} v_{j}(z)^{2}\right)$ converges to zero as $n, m \rightarrow \infty$. Hence $|F(z)|$ is in $L^{2}(H)$ for each $z>0$. That $\||F(z)|\|_{2}=$ $2^{1 / 2}\|U(z)\|_{2}$ follows by taking Wiener transforms in

$$
\begin{aligned}
E\left(|F(z)|^{2}\right) & =E\left(U(z)^{2}\right)+\sum_{j=1}^{\infty} E\left(v_{j}(z)^{2}\right) \\
& =E\left(|W(U)|^{2}\right)+E\left(\sum_{j=1}^{\infty}\left(B^{*}(\cdot), e_{j}\right)^{2}\left\|B^{*}(\cdot)\right\| \|^{-2}|W(U)|^{2}\right) \\
& =2 E\left(|W(U)|^{2}\right)=2 E\left(U^{2}\right) ;
\end{aligned}
$$

here the dominated convergence theorem is used as above to insure these equalities.

In what follows we shall denote $\partial / \partial z=A_{0}$ and the map $U(z) \rightarrow U(z+h)$ by $\left(T_{h} U\right)(z)$. Set $U(z)=v_{0}(z)$ and $A_{j} F(z)=\left(A_{j} v_{0}, A_{j} v_{1}, A_{j} v_{2}, \ldots\right)$ for $j=0,1,2,3, \ldots$ Then $\left|A_{j} F\right|$ and $\left|A_{j}^{2} F\right|$ are in $L^{2}(H)$ and $\left\|\left|A_{j} F\right|\right\|_{2} \leqq$ const $\|f\|_{2}$ and $\left\|\left|A_{j}^{2} F\right|\right\|_{2}$ $\leqq$ const $\|f\|_{2}$.

THEOREM 2. The notation is as established above. If $|F(z)|>0$ almost everywhere for each $z>0, \quad A_{j}|F(z)|=\left(F(z), A_{j} F(z)\right)|F(z)|^{-1}, j=0,1,2,3, \ldots$ The second derivative $A_{j}^{2}|F(z)|=\lim _{t \rightarrow 0} t^{-1}\left[T_{t B e_{j}} A_{j}|F(z)|-A_{j}|F(z)|\right]$ exists in measure. The sum $\sum_{j=0}^{\infty} A_{j}^{2}|F(z)|$ converges in measure and the sum is positive almost everywhere.

Proof. For brevity set $F(z)=F$ and $T_{t B e_{j}}=T_{t}$ for the computation of the derivatives.

Step I. We shall show that $\lim _{t \rightarrow 0}\left\|t^{-1}\left(T_{t}|F|-|F|\right)-|F|^{-1}\left(F, A_{j} F\right)\right\|_{2}=0$. Expand the integrand and consider

$$
I_{t}=E\left[|F|^{-2}\left(F, A_{j} F\right)^{2}-|F|^{-1}\left(F, A_{j} F\right) t^{-1}\left(T_{t}|F|-|F|\right)\right]
$$

and

$$
I I_{t}=E\left[t^{-2}\left(T_{t}|F|-|F|\right)^{2}-|F|^{-1}\left(F, A_{j} F\right) t^{-1}\left(T_{t}|F|-|F|\right)\right] .
$$

First note that $T_{t}|F|=\left|T_{t} F\right|$ almost everywhere. For if $F$ were a vector with finitely many nonzero components and if each component were a bounded continuous tame function, this equality would hold. The set of all vectors with finitely many nonzero components each of which is a bounded continuous tame function is dense in the set of all square integrable functions from $H$ to $l_{2}$. Hence the above equality holds.

$$
\begin{gathered}
I_{t}=E\left[|F|^{-1}\left(F, A_{j} F\right)\left(|F|^{-1}\left(F, A_{j} F\right)-t^{-1}\left(\left|T_{t} F\right|^{2}-|F|^{2}\right)\left(\left|T_{t} F\right|+|F|\right)^{-1}\right)\right] . \\
\left|T_{t} F\right|^{2}-|F|^{2}=\sum_{i=0}^{\infty}\left[T_{t} v_{i}+v_{i}\right]\left[t A_{j} v_{i}+o_{i}(t)\right]
\end{gathered}
$$

where $o_{i}(t)=\left(T_{t} v_{i}-v_{i}\right)-t A_{j} v_{i}$. Let $o(t)=\left(o_{0}(t), o_{1}(t), o_{2}(t), \ldots\right)$. Then

$$
E\left(\left|t^{-1} o(t)\right|^{2}\right)=E\left(\sum_{i=0}^{\infty}\left[t^{-1}\left(\exp i t / 2 F\left(B e_{j}\right)-1\right)-i / 2 F\left(B e_{j}\right)\right]^{2}\left|W\left(v_{i}\right)\right|^{2}\right)
$$


tends to zero by the dominated convergence theorem (using the fact that $v_{i}$ is a Poisson integral). Thus

$$
\begin{aligned}
I_{t}= & E\left[|F|^{-2}\left(F, A_{j} F\right)^{2}-\left(F, A_{j} F\right)|F|^{-1}\left(T_{t} F+F, A_{j} F\right)\left(\left|T_{t} F\right|+|F|\right)^{-1}\right] \\
& +E\left[\left(T_{t} F+F, t^{-1} o(t)\right)\left(\left|T_{t} F\right|+|F|\right)^{-1}\right] .
\end{aligned}
$$

Each integrand tends to zero in measure as $t \rightarrow 0$. By the Schwarz inequality, the first integrand is dominated by an integrable function which is independent of $t$; hence the first integral converges to zero as $t \rightarrow 0$. The second integral is dominated in absolute value by $\left\|t^{-1}|o(t)|\right\|_{2}$ by the Schwarz inequality and this tends to zero as $t \rightarrow 0$. Thus $\lim _{t \rightarrow 0} I_{t}=0$.

For $I I_{t}$ write

$$
\begin{aligned}
I I_{t}= & E\left[\left(\left|T_{t} F\right|+|F|\right)^{-2}\left(T_{t} F+F, A_{j} F\right)^{2}-|F|^{-1}\left(F, A_{j} F\right)\left(T_{t} F+F, A_{j} F\right)\left(\left|T_{t} F\right|+|F|\right)^{-1}\right] \\
& +2 E\left[\left(\left|T_{t} F\right|+|F|\right)^{-2}\left(T_{t} F+F, A_{j} F\right)\left(T_{t} F+F, t^{-1} o(t)\right)\right] \\
& +E\left[\left(T_{t} F+F, t^{-1} o(t)\right)^{2}\left(\left|T_{t} F\right|+|F|\right)^{-2}\right] \\
& -E\left[\left(|F|^{-1}\left(F, A_{j} F\right)\left(\left|T_{t} F\right|+|F|\right)^{-1}\left(T_{t} F+F, t^{-1} o(t)\right)\right]\right.
\end{aligned}
$$

where $o(t)$ is as above. The integrand in the first integral converges to zero in measure as $t$ tends to zero since $T_{t}$ is strongly continuous, and this integrand is dominated by $2\left|A_{j} F\right|^{2}$. So the first integral tends to zero with $t$. The second integral is dominated in absolute value by $2 E\left(\left|A_{j} F\right| t^{-1}|o(t)|\right) \leqq K\left\|t^{-1}|o(t)|\right\|_{2}$ which tends to zero with $t$. The third integral is dominated by $K\left\|t^{-1}|o(t)|\right\|_{2}^{2}$, as is the last integral, so that these tend to zero with $t$. We have calculated the first derivatives of $|F|$.

Step II. We show that $A_{j}^{2}|F|=|F|^{-1}\left|A_{j} F\right|^{2}+|F|^{-1}\left(F, A_{j}^{2} F\right)-|F|^{-3}\left(F, A_{j} F\right)^{2}$ and $t^{-1}\left(T_{t} A_{j}|F|-A_{j}|F|\right)$ converges in measure to $A_{j}^{2}|F|$ as $t$ tends to zero. (This difference quotient does not converge in $L^{2}(H)$. It is convenient, however, to continue to denote the second partial by $A_{j}^{2}$.)

$A_{j}|F|=|F|^{-1}\left(F, A_{j} F\right)$ and $T_{t} A_{j}|F|=\left|T_{t} F\right|^{-1}\left(T_{t} F, T_{t} A_{j} F\right)$ almost everywhere. Consider $|F| T_{t} A_{j}|F|$. Write $|F| T_{t} A_{j}|F|-|F| A_{j}|F|=a_{1}(t)+a_{2}(t)+a_{3}(t)$ where $a_{1}(t)=|F|\left|T_{t} F\right|^{-1}\left(T_{t} F, T_{t} A_{j} F\right)-\left(T_{t} F, T_{t} A_{j} F\right)=\left|T_{t} F\right|^{-1}\left(T_{t} F, T_{t} A_{j} F\right)\left(|F|-\left|T_{t} F\right|\right)$. By Step I, $|F|-\left|T_{t} F\right|=-|F|^{-1}\left(F, A_{j} F\right) t+o(t)$ where $\left\|t^{-1} o(t)\right\|_{2}$ tends to zero as $t$ tends to zero. So

$$
\begin{aligned}
& a_{1}(t)=t\left[-|F|^{-1}\left(F, A_{j} F\right)\right]\left|T_{t} F\right|^{-1}\left(T_{t} F, T_{t} A_{j} F\right)+o(t)\left|T_{t} F\right|^{-1}\left(T_{t} F, T_{t} A_{j} F\right) . \\
& a_{2}(t)=\left(T_{t} F, T_{t} A_{j} F\right)-\left(F, T_{t} A_{j} F\right)=t\left(A_{j} F, T_{t} A_{j} F\right)+\left(o(t), T_{t} A_{j} F\right)
\end{aligned}
$$

where $o(t)$ is as in Step I and $\left\|t^{-1}|o(t)|\right\|_{2}$ tends to zero with $t$.

Thus

$$
a_{3}(t)=\left(F, T_{t} A_{j} F\right)-\left(F, A_{j} F\right)=t\left(F, A_{j}^{2} F\right)+(F, o(t)) .
$$

$$
\begin{aligned}
\| t^{-1}|F| & \left(T_{t} A_{j}|F|-A_{j}|F|\right)-|F| A_{j}^{2}|F| \|_{1} \\
\leqq & \left\|t^{-1} a_{1}(t)+|F|^{-2}\left(F, A_{j} F\right)^{2}\right\|_{1}+\left\|t^{-1} a_{2}(t)-\left|A_{j} F\right|^{2}\right\|_{1}+\left\|t^{-1} a_{3}(t)-\left(F, A_{j}^{2} F\right)\right\|_{1} \\
\leqq & \left\||F|^{-2}\left(F, A_{j} F\right)^{2}-|F|^{-1}\left(F, A_{j} F\right)\left|T_{t} F\right|^{-1}\left(T_{t} F, T_{t} A_{j} F\right)\right\|_{1} \\
& +\left\|t^{-1} o(t)\left|T_{t} F\right|^{-1}\left(T_{t} F, T_{t} A_{j} F\right)\right\|_{1}+\left\|\left|A_{j} F\right|^{2}-\left(A_{j} F, T_{t} A_{j} F\right)\right\|_{1} \\
& +\left\|t^{-1}\left(A_{j} F, o(t)\right)\right\|_{1}+\left\|t^{-1}(F, o(t))\right\|_{1} .
\end{aligned}
$$


By the Schwarz inequality the first term on the right is dominated by

$$
K\left\|A_{j}|F|-T_{t} A_{j}|F|\right\|_{2}
$$

which converges to zero with $t$ because of the strong continuity of the $T_{t}$. The second term is dominated by $K\left\|t^{-1} o(t)\right\|_{2}$ and tends to zero with $t$. Similar arguments show that the other terms on the right tend to zero with $t$. This proves that $|F|\left[T_{t} A_{j}|F|-A_{j}|F|\right] / t$ converges in $L^{1}(H)$ to the desired quantity as $t$ tends to zero. Since $|F|>0$ almost everywhere for each $z>0, t^{-1}\left[T_{t} A_{j}|F|-A_{j}|F|\right]$ converges in measure to $|F|^{-1}\left|A_{j} F\right|^{2}+|F|^{-1}\left(F, A_{j}^{2} F\right)-|F|^{-3}\left(F, A_{j} F\right)^{2}$.

Step III. $\sum_{j=0}^{\infty} A_{j}^{2}|F|$ converges in measure and the sum is positive almost everywhere.

Notice first that by the conclusion of Step II, $|F| A_{j}^{2}|F| \geqq\left(F, A_{j}^{2} F\right)$ since $|F|^{-2}\left(F, A_{j} F\right)^{2} \leqq\left|A_{j} F\right|^{2}$.

$\sum_{j=0}^{\infty}\left|A_{j} F\right|^{2}$ converges in $L^{1}(H)$. For

$$
\begin{gathered}
E\left(\sum_{j=n}^{m}\left|A_{j} F\right|^{2}\right)=\sum_{j=n}^{m} E\left(\left|A_{j} F\right|^{2}\right)=\sum_{j=n}^{m} E\left(\sum_{i=0}^{\infty}\left(A_{j} v_{i}\right)^{2}\right) . \\
E\left(\left(A_{j} v_{i}\right)^{2}\right)=\frac{1}{4} E\left(\left(B^{*}(\cdot), e_{j}\right)^{2}\left(B^{*}(\cdot), e_{i}\right)^{2}\left\|B^{*}(\cdot)\right\|^{-2} \exp \left[-z\left\|B^{*}(\cdot)\right\|\right]|W(f)|^{2}\right)
\end{gathered}
$$

For $i=0$,

$$
E\left(\left(A_{j} v_{0}\right)^{2}\right)=\frac{1}{4} E\left(\left(B^{*}(\cdot), e_{j}\right)^{2} \exp \left[-z\left\|B^{*}(\cdot)\right\|\right]|W(f)|^{2}\right) .
$$

By the dominated convergence theorem, $E\left(\left|A_{j} F\right|^{2}\right)=\frac{1}{2} E\left(\left(B^{*}(\cdot), e_{j}\right)^{2}|W(U)|^{2}\right)$. As $m, n \rightarrow \infty, \sum_{j=n}^{m} E\left(\left|A_{j} F\right|^{2}\right)$ converges to zero since

$$
\sum_{j=n}^{m}\left(B^{*}(\cdot), e_{j}\right)^{2} \exp \left[-z\left\|B^{*}(\cdot)\right\|\right]
$$

converges boundedly to zero in measure.

Since $|F|^{-2}(F, A, F)^{2} \leqq\left|A_{j} F\right|^{2}, \sum_{j=0}^{\infty}|F|^{-2}\left(F, A_{j} F\right)^{2}$ converges in $L^{1}(H)$.

$\sum_{j=0}^{\infty}\left(F, A_{j}^{2} F\right)$ converges in $L^{1}(H)$. For

$$
\begin{gathered}
E\left(\left|\sum_{j=n}^{m}\left(F, A_{j}^{2} F\right)\right|\right)=E\left(\left|\left(F, \sum_{j=n}^{m} A_{j}^{2} F\right)\right|\right) \leqq E\left(|F|\left|\sum_{j=n}^{m} A_{j}^{2} F\right|\right) \\
\leqq\|\||F|\left\|_{2}\left|\sum_{j=n}^{m} A_{j}^{2} F\right|\right\|_{2} \\
\|\left.\left|\sum_{j=n}^{m} A_{j}^{2} F\right|\right|_{2} ^{2}=E\left(\sum_{i=0}^{\infty}\left(\sum_{j=n}^{m} A_{j}^{2} v_{i}\right)^{2}\right) \cdot E\left(\left(\sum_{j=n}^{m} A_{j}^{2} v_{i}\right)^{2}\right)=E\left(\sum_{j, k=n}^{m} A_{j}^{2} v_{i} A_{k}^{2} v_{i}\right) \\
=\frac{1}{16} E\left(\sum_{j, k=n}^{m}\left(B^{*}(\cdot), e_{j}\right)^{2}\left(B^{*}(\cdot), e_{k}\right)^{2}\left(B^{*}(\cdot), e_{i}\right)^{2}\left\|B^{*}(\cdot)\right\|^{-2} \exp \left[-z\left\|B^{*}(\cdot)\right\|\right]|W(f)|^{2}\right) \\
\text { when } i \geqq 1 \text { and } \\
E\left(\left(\sum_{j=n}^{m} A_{j}^{2} v_{0}\right)^{2}\right)=\frac{1}{16} E\left(\sum_{k, j=n}^{m}\left(B^{*}(\cdot), e_{j}\right)^{2}\left(B^{*}(\cdot), e_{k}\right)^{2} \exp \left[-z\left\|B^{*}(\cdot)\right\|\right]|W(f)|^{2}\right) .
\end{gathered}
$$


Thus

$$
\begin{gathered}
E\left(\left|\sum_{j=n}^{m} A_{j}^{2} F\right|^{2}\right)=\frac{1}{8} E\left(\sum_{j, k=n}^{m}\left(B^{*}(\cdot), e_{j}\right)^{2}\left(B^{*}(\cdot), e_{k}\right)^{2} \exp \left[-z\left\|B^{*}(\cdot)\right\|\right]|W(f)|^{2}\right) . \\
\sum_{j, k=n}^{m}\left(B^{*}(\cdot), e_{j}\right)^{2}\left(B^{*}(\cdot), e_{k}\right)^{2} \exp \left[-z\left\|B^{*}(\cdot)\right\|\right] \leqq\left\|B^{*}(\cdot)\right\|^{4} \exp \left[-z\left\|B^{*}(\cdot)\right\|\right]
\end{gathered}
$$

and this sum converges boundedly and in measure to zero as $n, m$ tend to infinity. Hence $\sum_{j=0}^{\infty}\left(F, A_{j} F\right)$ converges in $L^{1}(H)$.

Hence $\sum_{j=0}^{\infty}|F| A_{j}^{2}|F|$ converges in $L^{1}(H)$ and $\sum_{j=0}^{\infty} A_{j}^{2}|F|$ converges in measure.

Since $\sum_{j=0}^{\infty}|F| A_{j}^{2}|F| \geqq \sum_{j=0}^{\infty}\left(F, A_{j}^{2} F\right)$ and since each component $v$ of $F$ satisfies $\sum_{j=0}^{\infty} A_{j}^{2} v=0$, the right side of the last inequality converges to zero in $L^{1}(H)$. We know from above that $\sum_{j=0}^{\infty}\left(F, A_{j}^{2} F\right)$ converges in $L^{1}(H)$. Consider

and

$$
\sum_{j=0}^{n}\left(F, A_{j}^{2} F\right)=\left(F, \sum_{j=0}^{n} A_{j}^{2} F\right)
$$

$$
\begin{array}{r}
E\left(\left|F, \sum_{j=0}^{n} A_{j}^{2} F\right|\right) \leqq\||F|\|_{2}\left\|\sum_{j=0}^{n} A_{j}^{2} F \mid\right\|_{2} \\
\left\|\left|\sum_{j=0}^{n} A_{j}^{2} F\right|\right\|_{2}^{2}=E\left(\sum_{i=0}^{\infty}\left(\sum_{j=0}^{n}\left(A_{j}^{2} v_{i}\right)\right)^{2}\right) .
\end{array}
$$

Consider

$$
\begin{aligned}
E\left(\left(\sum_{j=0}^{n} A_{j}^{2} v_{i}\right)^{2}\right)=E\left[\left(\frac{\left\|B^{*}(\cdot)\right\|^{2}}{4}-\right.\right. & \left.\sum_{j=1}^{m} \frac{\left(B^{*}(\cdot), e_{j}\right)^{2}}{4}\right)^{2} \\
& \left.\times \exp \left[-z\left\|B^{*}(\cdot)\right\|\right]\left(B^{*}(\cdot), e_{i}\right)^{2}\left\|B^{*}(\cdot)\right\|^{-2}|W(f)|^{2}\right]
\end{aligned}
$$

for $i \geqq 1$ and

$$
E\left(\left(\sum_{j=0}^{n} A_{j}^{2} v_{0}\right)^{2}\right)=E\left[\left(\frac{\left\|B^{*}(\cdot)\right\|^{2}}{4}-\sum_{j=1}^{n}\left(B^{*}(\cdot), e_{j}\right)^{2}\right) \exp \left[-z\left\|B^{*}(\cdot)\right\|\right]|W(f)|^{2}\right] .
$$

By the dominated convergence theorem

$$
\begin{aligned}
\left\|\sum_{j=0}^{n} A_{j}^{2} F \mid\right\|_{2}^{2}= & 2 E\left[\left(\frac{\left\|B^{*}(\cdot)\right\|^{2}}{4}-\sum_{j=1}^{n} \frac{\left(B^{*}(\cdot), e_{j}\right)^{2}}{4}\right)^{2} \exp \left[-z\left\|B^{*}(\cdot)\right\|\right]|W(f)|^{2}\right] . \\
& \left(\frac{\left\|B^{*}(\cdot)\right\|^{2}}{4}-\sum_{j=1}^{n} \frac{\left(B^{*}(\cdot), e_{j}\right)^{2}}{4}\right)^{2} \exp \left[-z\left\|B^{*}(\cdot)\right\|\right]
\end{aligned}
$$

converges boundedly and in measure to zero. Hence $\left\|\left|\sum_{j=0}^{n} A_{j}^{2} F\right|\right\|_{2}^{2}$ converges to zero as $n$ tends to infinity. Hence $\sum_{j=0}^{\infty}\left(F, A_{j}^{2} F\right)$ converges to zero in $L^{1}(H)$ and $\sum_{j=0}^{\infty} A_{j}^{2}|F| \geqq 0$ almost everywhere.

REMARK 1. If $f$ is nonnegative and somewhere positive, $P_{z}(f)$ is positive almost everywhere and $|F|$ is positive almost everywhere. 
REMARK 2. An argument similar to that given above shows that if $G(z)$ $=\left((\partial / \partial z) U, A_{1} v_{1}, A_{2} v_{2}, A_{3} v_{3}, \ldots\right)$ and if $|G(z)|>0$ almost everywhere for $z>0$, the sum $\left(\partial^{2} / \partial z^{2}\right)|G(z)|+\sum_{j=1}^{\infty} A_{j}^{2}|G(z)|$ converges in measure and is positive almost everywhere.

\section{REFERENCES}

1. M. J. Fisher, Singular integral operators over a Hilbert space, Trans. Amer. Math. Soc. 131 (1968), 437-465.

2. - Tame singular integrals, Studia Math. (to appear).

3. L. Gross, Integration and nonlinear transformations in Hilbert space, Trans. Amer. Math. Soc. 94 (1960), 404-440.

4. - Harmonic analysis on Hilbert space, Mem. Amer. Math. Soc., No. 46 (1963).

5. - Abstract Wiener spaces, Proc. Fifth Berkeley Sympos. on Math. Stat. and Prob. (1965), Univ of California Press, Berkeley, Calif., 1967.

6. - Potential theory on Hilbert space, J. Functional Analysis 1 (1967), 123-181.

7. L. Hörmander, Estimates for translation invariant operators in $L^{p}$ spaces, Acta Math. 104 (1960), 93-139.

8. I. E. Segal, Tensor algebras over Hilbert spaces, Trans. Amer. Math. Soc. 81 (1956), $106-134$.

9. - Distributions in Hilbert space and canonical systems of operators, Trans. Amer. Math. Soc. 88 (1958), 12-41.

10. E. M. Stein and G. Weiss, On the theory of harmonic functions of several variables, Acta Math. 103 (1960), 25-62.

THE JOHNS HOPKINS UNIVERSITY, Baltimore, Maryland 